\title{
Éditorial
}

\section{De la nécessité d'une publication scientifique de haut niveau}

Les œuvres sont comme les hommes, elles se doivent de grandir.

L'idée, la volonté, le plaisir et quelques moyens permettent d'émerger du néant. Que de chemin parcouru depuis le numéro 1 de la Revue de la Société Française de Chirurgie buccale! Simple transcription écrite des communications présentées lors de nos congrès mais qui demandait déjà beaucoup de ténacité, cette revue s'est transformée par phases en une publication de qualité : «Médecine Buccale, Chirurgie Buccale».

Les articles répondant aux normes internationales sont apparus progressivement et plus régulièrement. Pour cela, chaque sociétaire, chaque responsable a offert le meilleur de lui-même. Chacun a participé, à sa mesure, par le biais de MBCB au développement de notre discipline. Chaque période s'est appuyée sur la précédente pour aller de l'avant. Certains ont contribué plus que d'autres, mais tous ont vécu ces phases de découragement qui par réaction sont sources de progrès.

Aujourd'hui, le progrès est manifeste mais insuffisant. Les exigences de la publication internationale sont nombreuses, et les règles bien connues. C'est pour cette raison que nous avons décidé de nous adosser à un éditeur scientifique professionnel : EDP Sciences, qui nous donne tous les moyens logistiques, stratégiques et numériques pour que nous puissions délivrer des publications de haut niveau tout en gardant l'âme et l'esprit de la Société Francophone de Médecine buccale et Chirurgie buccale. À la veille de la mise en place d'un internat qualifiant en chirurgie buccale, devant la qualité de nos cliniciens et la ténacité de nos chercheurs, nous ne pouvons plus douter du succès de cette entreprise. 